The Ffynnon Beuno and Cae Gwyn Caves.

I THINK it would be well for geologists and anthropologists to allow the age of the deposits and stone instruments found at these caves to remain an open question for the present. At present I have had no opportunity of seeing any papers on the subject, and I know of no opinions other than the one expressed by Dr. Hicks, NATURE, vol. .xxxvi. p. 599. I am how ever fairly well acquainted with the glacial deposits of North Wales and with Palitolithic implements, and I have seen the caves and the tools found at and in them. My quite unbiased opinion is and will so remain,-unless I get very convincing proof to the contrary,- - that the drift at the caves has been withont doubt relaid; and is no more a true glacial deposit than the valley gravels of the Thames. As for the tools-one in the British Museum (South Kensington), and one in Denbighshirethey belong to the very latest of Palrolithic times, and might be passed for Neolithic; the Denbighshire example seen by me is a knife-flake with fine secondary chipping up one edge.

Dunstable. WORTHINGTON G. SMITH.

\section{SYNTHESIS OF GLUCOSE.}

$\mathrm{A}^{\mathrm{N}}$ THER important acquisition to our store of knowledge has recently been made. Glucose, commonly called grape-sugar, has been artificially prepared by Drs. Emil Fischer and Julius Tafel in the chemical laboratory of the University of Würzburg. This happy achievement, which is announced in the number of the Berichte just received, is one which has long been looked forward to, and which cannot fail to give deep satisfaction in chenical circles all over the world. As is generally the case in syntheses of this description, not only has the sugar itself been actually prepared, but, what is at least quite as important, considerable light has been thrown upon that much-discussed question - the constitution of sugars. A most remarkable, and yet only to be expected, attribute of this artificial sugar is that it is found to be entirely incapable of rotating a beam of polarized light. As is well known, there are several naturally-occurring varieties of glucose, all of which may be expressed by the same empirical constitution $\mathrm{C}_{6} \mathrm{H}_{12} \mathrm{O}_{6}$, and all possessing the power of rotating the plane of polarization: dextrose, or grape-sugar, the best-known of these varieties, as its name implies deviates the plane of polarization to the right, as do several other less important varieties; while lævulose, or fruit-sugar, rotates the plare to the left. But in artificially preparing a glucose of the composition $\mathrm{C}_{6} \mathrm{H}_{12} \mathrm{O}_{6}$ there is just as much tendency for one kind to be formed as another, and the probability is that both dextro and lævo are simultaneously formed, and thus neutralize each other, producing a totally inactive mixture. It may be that, as in the case of racemic acid, the two kinds are formed side by side and neutralize each other in the solution ; or it may even be that, as is the case with truly inactive tartaric acid, there is a true reutralization within the molecule itself; which of these bypotheses is correct is a question for further work to decide.

The substance employed as the base of operations was acrolein, $\mathrm{CH}_{2}=\mathrm{CH} \quad \mathrm{CHO}$, the aldehyde derived by oxidation of allyl alcohol. The acrolein was first converted to its dibromide, $\mathrm{CH}_{2} \mathrm{Br}$. $\mathrm{CHBr}$. $\mathrm{CHO}$, which was then treated with cold baryta water, whereupon the bromine was removed by the barium leaving the artificial sugar in solution. The real difficulty was found to be in the isolation of the sugar, but this was eventually overcome by the use of phenyl hydrazine, $\mathrm{C}_{6} \mathrm{H}_{5} . \mathrm{NH} . \mathrm{NH}_{2}$, which forms a hydrazine compound of the formula $\mathrm{C}_{18} \mathrm{H}_{22} \mathrm{~N}_{4} \mathrm{O}_{4}$ with the new sugar, very similar to the compounds formed by phenyl hydrazine with ordinary dextrose and lævulose. This phenyl hydrazine compound was then found to yield by reduction a base $\mathrm{C}_{6} \mathrm{H}_{13} \mathrm{NO}_{5}$, which, on treatment with nitrous acid, parted with its nitrogen and left a syrupy substance, possessing all the properties of sugars, and distinguished only from ordinary grape-sugar by its optical inactivity.
The actual operations were performed briefly as follows :-

Seventy-five grammes of pure crystallized barium hydrate were dissolved in a little over a litre of water, and 50 grammes of previously redistilled acroleïn dibromide added drop by drop, the flask being continuously agitatec, surrounced by ice-cold water, for about an hour. In a similar manner eight successive quantities were treated until in all about 400 grammes of acroleïn dibromide had been converted into sugar. These eight separate portions were then mixed, slightly acidified with sulphuric acid, and the barium precipitated with a solution of sodium sulphate. After removal of all the barium by filtration the solution was neutralized with soda and evaporated down to $I \frac{1}{2}$ litres. On cooling, a solution of 50 grammes of the hydrochloride of phenyl hydrazine and 50 grammes of crystallized sodium acetate in 100 cubic centimetres of water were added; after standing twelve hours a reddishbrown resin separated out and was removed by filtration. I 50 grammes more of phenyl hydrazine hydrochloride and the same quantity of sodium acetate were then added, and the solution warmed upon a water-bath; after again standing some time the solution became. turbid, and in course of four hours a dark-coloured precipitate, partly crystalline and parlly resinous, separated out. After washing and drying, and subsequent agitation with ether and trituration with alcohol to remove organic impurities, and extraction of the inorganic salts by hot water, the phenyl hydrazine compound was finally isolated.

Analysis of the recrystallized compound indicates that its composition is $\mathrm{C}_{18} \mathrm{H}_{22} \mathrm{~N}_{4} \mathrm{O}_{4}$, and its properties are very similar to those of the phenyl hydrazine compound of ordinary grape-sugar, the melting-points of the two bodies being identical, $205^{\circ} \mathrm{C}$. It is almost insoluble in water, ether, and benzene, and only with difficulty soluble in hot alcohol ; it is more soluble in glacial acetic acid, but the solution soon becomes dark red. It crystallizes from hot alcohol in pretty little prism aggregates, while the ordinary grape-sugar compound crystallizes in spherical aggregates of fine needles. It is further distinguished from the latter compound inasmuch as a layer 20 cubic centimetres thick, is without action upon a beam of polarized light.

When reduced by means of zinc dust and acetic acid, a base was produced analogous to the one formed by the reduction of the corresponding phenyl hydrazine compound of grape-sugar. This base was difficult to isolate, owing to the non-crystallizable nature of its acetate; the fact was fortunately discovered, however, that its oxalate was crystalline, and readily obtained pure. Hence its analysis has been effected, and the numbers found point to the composition $\left(\mathrm{C}_{6} \mathrm{H}_{13} \mathrm{NO}_{5}\right)_{2} \cdot \mathrm{C}_{22} \mathrm{H}_{2} \mathrm{O}_{4}$. This base reduces Fehling's solution strongly on warming, and with phenyl hydrazine regenerates the parent compound; but, once again, is optically inactive.

Finally, by the action of nitrous acid, nitrogen at once began to be evolved, and when the evolution ceased the liquid was neutralized with soda, evaporated in vacuo, and the residue extracted with alcohol. On evaporation of the alcohol the sugar was left as a bright brown syrup, free from nitrogen and ash, of sweet taste, and capable of instantly reducing Fehling's solution.

Up to the present time two hypotheses as to the constitution of sugars have pretty evenly balanced each other. According to one, sugars are considered, in virtue of their power of reducing ammoniacal silver solutions, as aldehydes containing also alcohol groups; on these lines grape-sugar would be formulated, $\mathrm{CH}_{2} \mathrm{OH}-$. $(\mathrm{CHOH})_{4}-\mathrm{CHO}$. But it has since been shown that the property of reducing ammoniacal silver solutions is not confined to aldehydes, for the series of bodies known as ketone alcohols also possess it ; hence grapesugar may also be written $\mathrm{CH}_{2} \mathrm{OH}-(\mathrm{CHOH})_{3}-\mathrm{CO}-$ 
$\mathrm{CH}_{2} \mathrm{OH}$. Both theories account for most of the hitherto known reactions of the glucoses, hence the matter has remained an open question. Drs. Fischer and Tafel, however, consider that their synthesis from acroleïn, which is itself an aldehyde, points to the probability of the former hypothesis being the correct one. The action of baryta water upon the dibromide evidently causes a simple exchange of bromine for hydroxyl, and the first product of the reaction is almost as certainly glycerine aldehyde, $\mathrm{CH}_{2} \mathrm{OH}-\mathrm{CHOH}-\mathrm{CHO}$. This latter substance, however, appears to polymerize at once under the influence of the baryta water into sugar, two molecules of glycerine aldehyde uniting to form a molecule of glucose.

In consideration of the fact of its derivation from acroleïn, the name acrose has been applied to the sugar which has been, with so much skill and steady determination, synthetically formed and isolated ; and there can be no doubt that this name will stand as a memento of the progress made in organic chemistry during the year 1887 .

A. E. TuTTON.

\section{MODERN VIEWS OF ELECTRICITY.' \\ PART II.-CURRENT ElECTRICITY (continued). IV. \\ Electrical Inertia.}

$\mathrm{R}^{\mathrm{E}}$ TURNING now to the general case of conduction, without regard to the special manner of it, we must notice that, if a current of electricity is anvthing of the nature of a material flow, there would probably be a certain amount of inertia connected with it, so that to start a current the stoppage of a current would also have either to be gradual or else violent. It is well known that if water is stagnant in a pipe it cannot be quite suddenly set in motion; and again, if it be in motion, it can only be suddenly stopped by the exercise of very considerable force, which jars and sometimes bursts the pipe. This impetus of running water is utilized in the water-ram. It must naturally occur, therefore, to ask whether any analogous phenomena are experienced with electricity; and the answer is, they certainly are. A current does not start instantaneously: it takes a certain time-often very short-to rise to its full strength; and when started it tends to persist, so that if its circuit be suddenly broken, it refuses to stop quite suddenly, and bursts through the introduced insulating partition with violence and heat. It is this ram or impetus of the electric current which causes the spark seen on breaking a circuit; and the more sudden the breakage the more violent is the spark apt to be.

The two effects - the delay at making circuit, and the momentum at breaking circuit-used to be called "extracurrent" effects, but they are now more commonly spoken of as manifestations of "self-induction."

We shall understand them better directly; meanwhile they appear to be direct consequences of the inertia of electricity; and certainly if electricity were a fluid possessing inertia it would behave to a superficial observer just in this way.

But if an electric current really possessed inertia, as a stream of water does, it would exhibit itself not only by these effects but also mechanically. A conducting coil delicately suspended might experience a rotary kick every time a current was started or stopped in it ; and if a steady current were maintained in such a coil it should behave like a top or gyrostat, and resist any force tending to deflect its plane.

Clerk Maxwell has carefully looked for this latter form of momentum effect, and found none. One may say, in fact, that nothing like momentum has yet been observed in an electric current by any mechanical mode of examination. A coil or whirl of electricity does not behave in the least like a top.

Does this prove that a current has no momentum? By no means necessarily so. It might be taken as suggesting that an electric current consists really of two equal flows in contrary directions, so that mechanically they neutralize one another completely, while electrically-i.e. in the phenomena of self-induction or extra-current-they add their effects. Or it may mean merely that the momentum is too minute to be so observed. Or, again, the whole thing-the appearance of inertia in some experiments and the absence of it in others-may have to be explained in some altogether less simple manner, to which we will proceed to lead up.

\section{Condition of the Medium near a Circuit.}

So far we have considered the flow of electricity as a phenomenon occurring solely inside conductors; just as the flow of water is a phenomenon occurring solely inside pipes. But a number of remarkable facts are known which completely negative this view of the matter. Something is no doubt passing along conductors when a current flows, but the disturbance is not confined to the conductor; on the contrary, it spreads more or less through all surrounding space.

The facts which prove this have necessarily no hydraulic analogue but must be treated suorum generum, and they are as follows:-

(I) A compass needle anywhere near an electric current is permanently deflected so long as the current lasts.

(2) Two electric currents attract or repel one another, according as they are in the same or opposite directions.

(3) A circuit in which a current is flowing tends to enlarge itself so as to inclose the greatest possible area.

(4) A circuit conveying a current in a magnetic field tends either to enlarge or to shrink or to turn half round according to the aspect it presents to the field.

(5) Conductors in the neighbourhood of an electric circuit experience momentary electric disturbances every time the current is started or stopped or varied in strength.

(6) The same thing happens even with a steady current if the distance between it and a conductor is made to vary.

(7) The effects of self-induction, or extra-currents, can be almost abolished by twisting the covered wire conveying the current closely on itself, or even by laying the direct and return wire side by side ; whereas they may be intensified by making the circuit inclose a large area, more by coiling it up tightly into close coil, and still more by putting a piece of iron inside the coil so formed.

Nothing like any of these effects is observable with currents of water; and they prove that the phenomena of the current, so far from being confined to the wire, spread out into space and affect bodies at a considerable distance.

Nearly all this class of phenomena were discovered by Ampere and by Faraday, and were called by the latter "current-induction." According to his view the dielectric medium round a conducting circuit is strained, and subject to stresses, just as is the same medium round an electrically charged body. The one is called an electrostatic strain, the other an electro-magnetic or electrokinetic strain.

But whereas electrostatic phenomena occur solely in the medium-conductors being mere breaks in it, interrupters of its continuity, at whose surface charge-effects occur but whose substance is completely screened from disturbance-that is not the case with electro-kinetic phenomena. It would be just as erroneous to conceive electro-kinetic phenomena as occurring solely in the insulating medium as it would be to think of them as occurring solely in the conducting wires. The fact is, they occur in 